\title{
A STUDY OF COLLAPSE SUSCEPTIBILITY AND RESISTANCE OF LOADED CABLE-SUPPORTED PIPE STRUCTURE SUBJECT TO A SUDDEN BREAK OF CABLE MEMBER
}

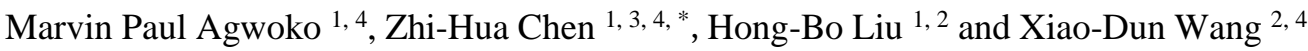 \\ ${ }^{1}$ State Key Laboratory of Hydraulic Engineering Simulation and Safety, Tianjin University, Tianjin 300072, China \\ ${ }^{2}$ Department of Civil Engineering, Hebei University of Engineering, Handan 056000, China \\ ${ }^{3}$ Key Laboratory of Coast Civil Structure Safety of China Ministry of Education, Tianjin University, Tianjin 300072, China \\ ${ }^{4}$ Department of Civil Engineering, Tianjin University, Tianjin 300072, China \\ * (Corresponding author: E-mail: zhchen@tju.edu.cn)
}

\section{A B S T RA C T}

Cable-supported pipe system (CSPS) provides a suitable system of structure for meeting the stringent structural requirements of pipeline bridges. However, due to a composite action of cable with truss and pipe members, the sudden failure of its structural member may lead to undesired vibratory response and collapse. The occurrence of a sudden break of the CSPS structural member is characterized by spontaneous dynamics and internal force rearrangement. The present study aims to investigate parametrically the collapse susceptibility and resistance of scaled down CSPS model in the event of a sudden break of the cable member by combined experimental and numerical procedures. The displacement of the structure, the pattern of internal force rearrangement, and dynamic responses were comparatively evaluated. Experimental results depict imminent cable failure under load and attendant dynamic response, but without a total collapse of the CSPS structure. Critical members causing large dynamic response amplitudes were identified and the mitigation of collapse was evaluated. Dynamic increasing factor (DIF) methods was utilized for the evaluation of the dynamic response of the sudden cable break resulting from the pattern of responses between the cable members and the rest of the CSPS structure. Comparison with provisions in other studies shows higher values DIF of the CSPS cable members which led to proposed evaluation using dynamic factor (DF). Thus, the dynamic factors for the sudden break of various cable members along the span and the errors were also estimated considering the parametric of design variables which will enable easy utilization during the structural process of CSPS

\section{A R T I C L E H I S T O R Y}

$\begin{array}{ll}\text { Received: } & \text { 23 June } 2020 \\ \text { Revised: } & \text { 3 March 2021 } \\ \text { Accepted: } & \text { 13 March 2021 }\end{array}$

\section{KEYWOR D S}

Cable-supported pipe system; Sudden break of cable; CSPS vibratory response; Collapse resistance;

Dynamic increasing factor; Dynamic factor

\section{Introduction}

Pipe structures are widely used as fluid conveyance systems which occasionally require bridging over a large space. Application can be found in the field requirement of agriculture, such as irrigation, application of fertiliser and pesticide [1], and other requirements in terminal buildings, onshore and offshore structures [2]. To meet responsive large span for elevated pipe structures, cable-supported pipe system (CSPS) is suggested as an alternative to the pipe bridge structure [3]. The CSPS structural composition is as shown in
Fig. 1(a) and the perspective view the typical model in Fig. 1(b). The CSPS is referred to as an innovative system of cable-truss structure possessing the combined arrangement of lattice trusses and cable to hold conveyance pipelines above the field. Thus, it offers the advantages of the spatial capability, economical and easy constructability when pipes are to be connected in a large space. These advantage confer great value of its application in irrigation structures such as the Centre pivot system [4]. However, a CSPS structural member may be at risk of collapse due to the sudden break of one or more cable members.

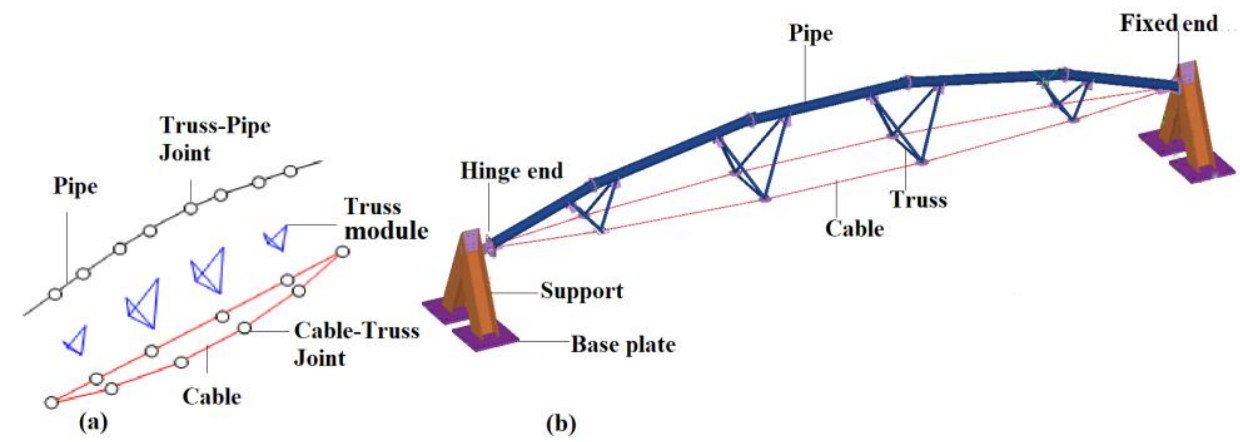

Fig. 1 Description of typical experimental model of Cable-supported pipe structure: (a) Schematic of the composition of the CSPS and (b) A typical CSPS experimental model

The sudden break of CSPS structural member may be encountered due to unforeseen design requirements, accident or natural disaster which consequently triggers vibratory responses and load redistribution on the structure. Consequently, the failure of more members and the imminent collapse of the CSPS structure may be encountered. It is thus crucial to consider the safety and robust behaviour of the structure during its service life in the event of sudden loss of member.

In the past, research mainly focused on cable-stayed bridges [5-7], cable roofs [8], and transmission line towers [9], among others. The study presented by Mozos deliberated on the internal force response of a cable-stayed bridge with a cable's sudden break and the influence of a single cable failure on the dynamic response of the entire bridge, $[5,6,10]$. Provision for the dynamic increasing factor for stay bridges is as provided by the post tension institute (PTI) [11]. The study was limited to shared influence between the rest bridge cables during the rupturing process. In Zhou, a time-progressive nonlinear dynamic analysis approach and a framework of nonlinear dynamic simulation to investigate the influence of an abrupt cable-breakage in a cable-stayed bridge and evaluate the safety of a cable-stayed bridge under cable loss scenarios was proposed [12-14]. With the increase in the application of cables as support for structures in recent years, more studies are conducted on suspendomes and cable-dome in China. Wang and Chen studied loaded suspendome subjected to sudden cable failure through numerical and experimental procedures and were able to propose indices that could be utilized for the structural design process $[8,15]$. 
Some design guides are obtainable in Europe and the United States codes for the mitigation of the progressive collapse of buildings. Such design guides include the design code of British Building Regulations (Office of the Deputy Prime Minister 2004), BS5950 [16], the procedures stipulated by Department of Defense (DoD) and the General Services Administration (GSA 2003) [17, 18]. Other institutions such as the Federal Emergency Management Agency (FEMA) [19] and the National Institute of Standards and Technology (NIST) [20] also provided general design guides, which necessitate provision of adequate redundancy and resilience of steel-framed structural systems [21, 22]. However, most of the design guides paid more attention to buildings leaving gap to be filled for the prevention of progressive collapse of CSPS structure.

This study focuses on the investigation of collapse susceptibility and mitigation strategy of CSPS structure. Thus, an experimental procedure is employed to determine the failure mode of the CSPS models with different boundary conditions. Owing to capital demands offered by the experimental processes, more parameteric studies are conducted by numercal processes after the validation. The experimental evaluation of a collapse resistance of a scaled down $8 \mathrm{~m}$ long loaded CSPS models were firstly achieved using loading platform setup in the laboratory. Then, explicit dynamic evaluation of the finite element (FE models) using finite-element analysis program ANSYS/LS-DYNA and validation with experimental data. Finally, the results of vibratory responses considering various parameters are evaluated and compared with the recommendation by PTI and possible adoption in the design process of the CSPS structures.

\section{Development of Models and procedures}

\subsection{Model geometric and design parameters}

In order to present typical structure, reference is made to other cable-supported structures having similar geometric configuration, including beam-string structure and cable-struts structures (CSS) [23]. Previous work is also available in the study of dynamic characteristics of the CSPS [3].

The mid-span depth is considered indispensable design parameter of systems. In Chinese code for design of steel structures (GB50017-2017), the limit of deformation is specified based on deflection-span ratio of beam-string 1/400 [24]. Consequently, some geometric parameters are presented in Fig. 2. The parameters can be identified as hogging depth of pipe system $(d)$ and sagging depth of cable supports $(f)$, depth at mid-span $(h)$ and the lateral width of cable support from pipe plane (w) as shown in Fig. 1(a) and (b) respectively. The width is related to the depth parameter and tied to $0.75 h \leq w \leq h$, upon which the lateral displacement is controlled.

Fig. 2(c) demonstrates the influence of loading due to both cable pretension and a vertical load of the CSPS structure. The mid-span deflection, lateral and longitudinal displacement on application of load are represented by $\delta z, \delta y$ and $\delta x$. In addition to the deflection, the study considers the effect of the structural member parameters on the CSPS model. Fig. 3 shows the node nomenclature, the cross-section of the CSPS model and the cross-section parameters of the structural members.

In Fig. 3, a CSPS model consisting of 4 truss modules is presented. The structural members consist of nine pipe segments labeled P1 to P9, twenty truss members labeled $\mathrm{T} 1$ to $\mathrm{T} 20$ and the cable members labeled $\mathrm{C} 11$ to $\mathrm{C} 15$ and $\mathrm{C} 21$ to $\mathrm{C} 25$ in respective runs. The parameter $t$ - $x p$ defines the pipe thickness, $r-x c$ the cable radius and $t-x t$ the truss member thickness. Six CSPS experimental models presented in Fig. 4 are considered for the evaluation. Consequently, each experimental model has the following similar design parameters: $L=8 \mathrm{~m}, r-x c=5.35 \mathrm{~mm}, t-x t=3 \mathrm{~mm}$ and $t-x p=3 \mathrm{~mm}$ were selected. The effect of the variables $r-x c, t-x t, t-x p$ were thus evaluated parametrically using finite element analysis (FEA). Details of the experimental model is presented in Table 1. Double tube pipe member is included as additional pipe parameter in the event of hydraulic modification. Thus, the study includes the evaluation of 3 single tube (ST) and 3 double tube (DT) CSPS models. (a)

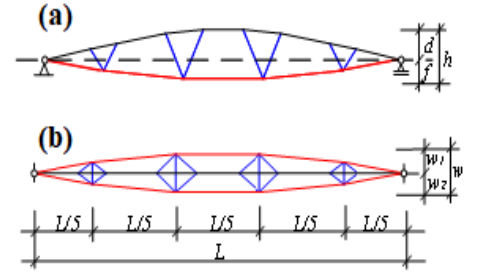
$0 \leq \frac{d}{L} \leq 0.05, \quad 0 \leq \frac{f}{L} \leq 0.05$
$h=d+f$

$w_{1}=w_{2}$
$w=w_{1}+w_{2}$
$0 \leq w \leq h$

(c)
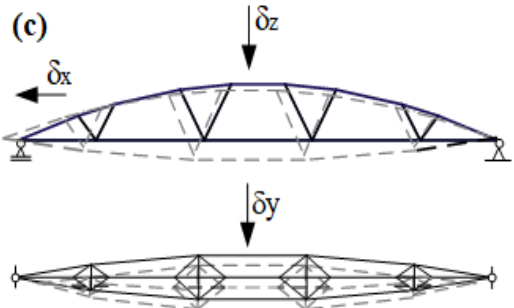

Fig. 2 CSPS geometric parameters: (a) Sketch of vertical profile, (b) Layout and (c) Deformation under load and (d) Lateral deformation

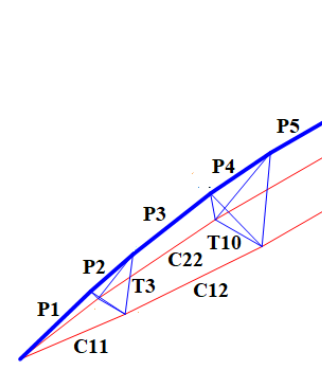

CISS model

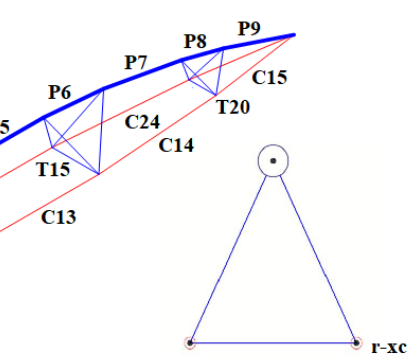

Cross-section

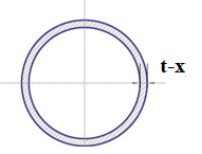

Pipe section
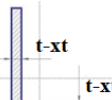

Truss section
Fig. 3 CSPS model with four truss modules and single tube pipe member

The section properties of the members consist: $114 \mathrm{~mm}$ outer diameter of pipe and $3 \mathrm{~mm}$ thickness, $36 \mathrm{~mm} \times 36 \mathrm{~mm} \times 3 \mathrm{~mm}$ angle cross-section of truss, and $10.8 \mathrm{~mm}$ cross-section diameter of cable. All materials are made of Chinese steel grade Q345 with tensile test results as follows: Modulus of elasticity, $E=199 \mathrm{MPa}$, Density, $\rho=7850 \mathrm{~kg} / \mathrm{m}^{3}$ and poison's ratio, $v=0.3$.

\subsection{Experimental setup}

The experimental setup includes the loading frame and the static load and reaction beams, as well as loading pad and the CSPS model as shown in Fig. 5. Detailed description of the method of applying the static load and measurements are presented in separate study with reference to works of Chen [15] and Wang [25] on suspendome structure. However, brief overview is provided hereunder.
The CSPS model is supported at one end with a fixed reaction support and at the other end with a roller reaction support both anchored onto a firm test platform at two ends. As depicted in Fig. 5, the experimental platform is made of adjustable loading frame and reaction beam placed above the loading positions of the CSPS models.

The first step of the experiment includes loading of the structure to simulate a static response. Point loads were applied at loading positions depicted in Fig. 5 with the aid of a pipe clamp loading pad (PCLP) through plunger rods that were connected to the top reaction beam. Details of the PCLP are provided in separate work on the research on the CSPS. In the next step of experiment, the CSPS is loaded until sudden break of a member is achieved. In some studies, the member breakage are undertaking using a break device at predefined structural member, and has been used successfully in the progressive collapse assessments of truss and steel dome model structures [8, 26]. In this study, the breaking of cable member was allowed to take place under load considering the resistance of the cable member joint (turnbuckle joint). This allow the advantage of a random selection of the breaking of cable member and allows the determination of the member removal time.

As earlier introduced, a sudden break of a cable member from a structure activates a time dependent vibratory response with consequent displacement and internal force redistribution. Observation of the vibratory time history was achieved with the aid of video records. The video was evaluated to extract time of response. The displacement and force are measured using displacement and strain data acquisition system.

\subsection{Numerical}

The 3-dimensional nonlinear model of the CSPS models presented in Fig. 
4 are developed in ANSYS Workbench. At first, an implicit model of the structure was developed in which BEAM188 element was applied to the pipe members and LINK180 element to the truss and cable members. Cable pretension was applied to reflect the measured tension on the cable members during the setup of the experimental model. Since tension rod were used as cable members, the influence of cable pretension was minimal, however, maximum pretension value was $0.9 \mathrm{KN}$. Thus, zero strain was considered in the cable member. The joints were considered as pin as adopted from the experimental models connection. To simplify the model, the support frames are replaced with fixed end and roller support. The tested modulus of elasticity of steel during the experiment was applied to the numerical model.

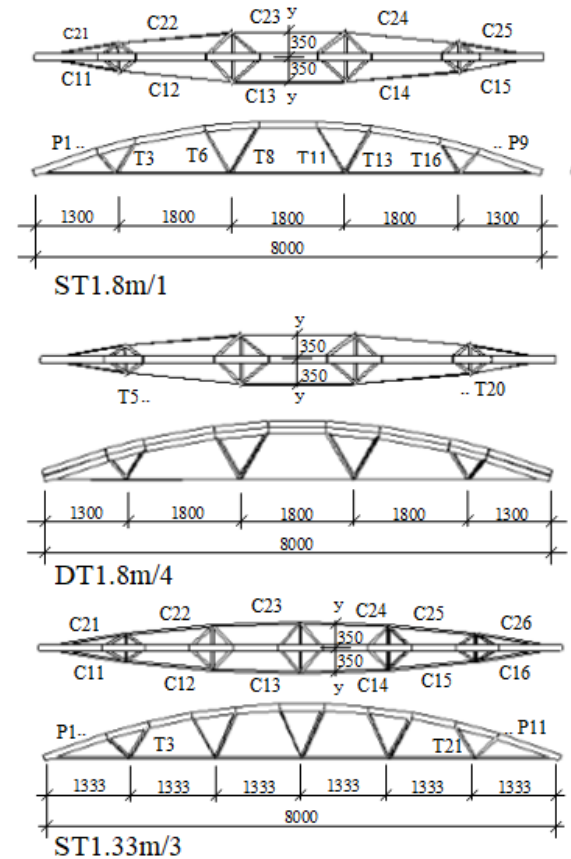

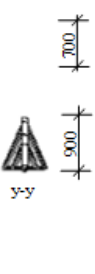

茟
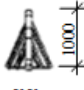
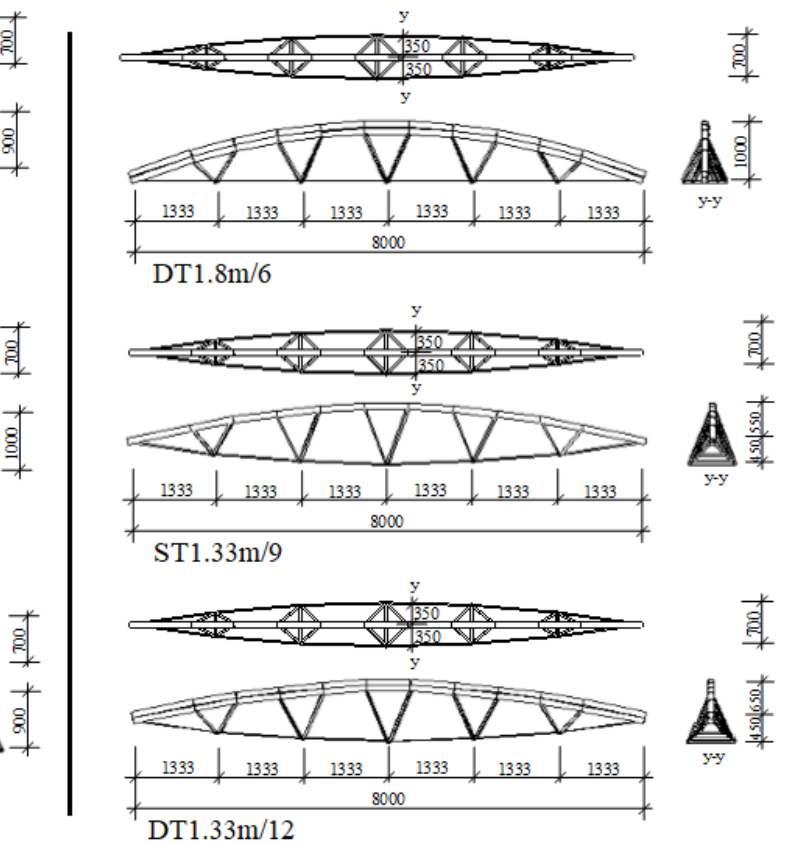

Fig. 4 Geometry diagram of the CSPS experimental models with both single and double tube pipe

Table 1

CSPS model parameters

\begin{tabular}{|c|c|c|c|c|c|c|c|c|}
\hline Model Ref. No. & Weight (kg) & $\begin{array}{l}\text { Truss max. spacing } \\
(\mathrm{mm})\end{array}$ & $\mathrm{d}(\mathrm{mm})$ & $\mathrm{f}(\mathrm{mm})$ & $\mathrm{h}(\mathrm{mm})$ & $\mathrm{h} / \mathrm{L}$ & No. of truss & No. of cable \\
\hline $\mathrm{ST} 1.8 \mathrm{~m} / 1$ & 102.2 & 1800 & 900 & 0 & 900 & 0.1125 & 20 & 10 \\
\hline DT $1.8 \mathrm{~m} / 4$ & 171.7 & 1800 & 1000 & 0 & 1000 & 0.1250 & 20 & 10 \\
\hline $\mathrm{ST} 1.33 \mathrm{~m} / 3$ & 106.9 & 1333 & 900 & 0 & 900 & 0.1125 & 25 & 12 \\
\hline DT $1.3 \mathrm{~m} / 6$ & 176.7 & 1800 & 1000 & 0 & 1000 & 0.1250 & 20 & 10 \\
\hline $\mathrm{ST} 1.33 \mathrm{~m} / 9$ & 108.9 & 1333 & 550 & 450 & 1000 & 0.1250 & 25 & 12 \\
\hline DT $1.33 \mathrm{~m} / 12$ & 177.2 & 1333 & 650 & 450 & 1100 & 0.1375 & 25 & 12 \\
\hline
\end{tabular}

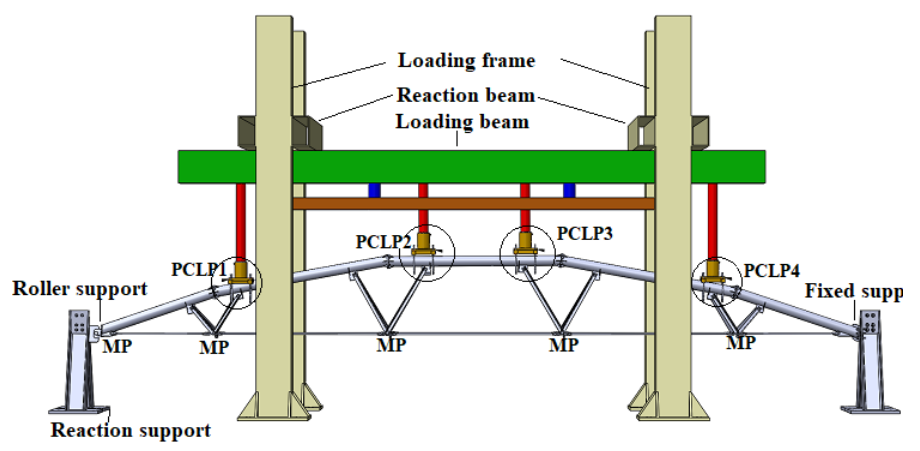

Fig. 5 Experimental setup

The general procedure for the analysis and design of structure against sudden failure of member and progressive collapse of structure are provided in design guidance of the General service Administration (GSA) and Unified Facilities Criteria (UFC) $[17,18]$. The proposed procedure by GSA is based on the Alternate Path Method (APM). The procedure places the condition of element removal scenarios for the analysis of structural response in the event of damage or sudden break of members, while the UFC procedure found on the provisions of GSA is a performance-based design approach. These procedures stipulate a set of analyses involving static nonlinear and nonlinear dynamic in the time integration steps, of which the process is tedious.
The explicit procedures of ANSYS/LS-DYNA [27] provides a facilitated and more concise approach for the analysis of structure in the occasion of sudden failure of a member. It also facilitates a process of application of different failure loads, including tie-break, blast and collision. The tie-break is defined as plastic kinematic model, which is dependent on strain for its failure [28]. Thus, when the member is strained beyond the defined limit, the member suddenly breaks from the rest of the structural model.

This study adopted the explicit procedure in which the implicit model was then changed to the explicit framework where the pipe members are model as BEAM161, the truss members as LINK160 and cable members as LINK167 elements. The analysis setting was made by applying the observed time duration from the experimental study and pre-processed in the Workbench interface while enabling post-processing of results in the LS-DYNA interface.

\section{Verification of the model}

\subsection{The member break phenomenon}

The setup of each CSPS model and the broken cable member are shown in Fig. 6. As earlier noted, the experimental setup is intended to have the failed cable member selected at random during the loading test. This is aided with the use of a single bolt connection to the cable-truss joint. The cable member joints are made of turnbuckle to provide resistance against shearing of the connecting bolt or sliding of the tension rod out of the turnbuckle through the tension control heads as depicted in Fig. 7(a). After the static loading reached the ultimate limit, the weakest joint fails by shearing of the 
connecting bolt/ buckling of the turnbuckle as shown in Fig. 7(b) or by the sliding of the tension rod out of the turnbuckle as shown in Fig. 7(c).

The experiment offered the advantage of identifying the structural member with the higher chance of sudden break when the structure is loaded. It was observed that the sudden break of the cable member of the CSPS resulted to transient response, large displacement and internal force redistribution. According to the arrangement of the models in Fig. 6(a), the broken cable members in models ST $1.8 \mathrm{~m} / 1$, DT $1.8 \mathrm{~m} / 4, \mathrm{ST} 1.33 \mathrm{~m} / 3$,
DT1.33m/6, ST1.33m/9 and DT1.33m/12 are C13, C13, C14, C16, C15 and C13, respectively. However, the structure exhibited some residual capacity to withstand the collapse of the extire structure. The observed vibratory response of all the models was in the time limit of 1s. Since the experimental process is tedious and economically unrealistic to excute more model within the available funds, further determination of the effect of a sudden break of each member were achieved through numerical procedures after the validation.

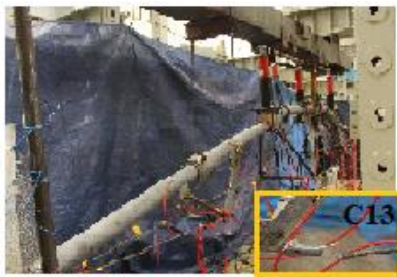

$\mathrm{ST} 1.8 \mathrm{~m} / 1$

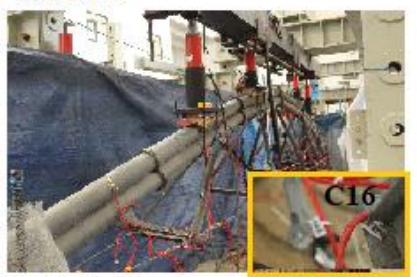

DT $1.33 \mathrm{~m} / 6$

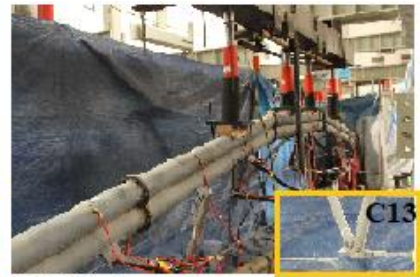

DT1.8m/4

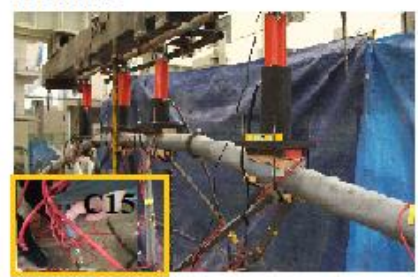

$\mathrm{ST} 1.33 \mathrm{~m} / 9$

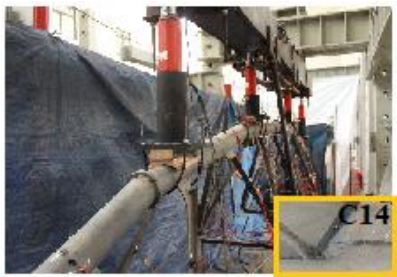

$\mathrm{ST} 1.33 \mathrm{~m} / 3$

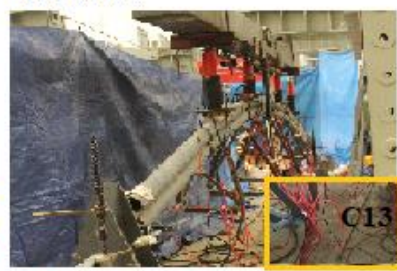

DT1.33m/12

Fig. 6 Loaded CSPS experimental models with broken members inset

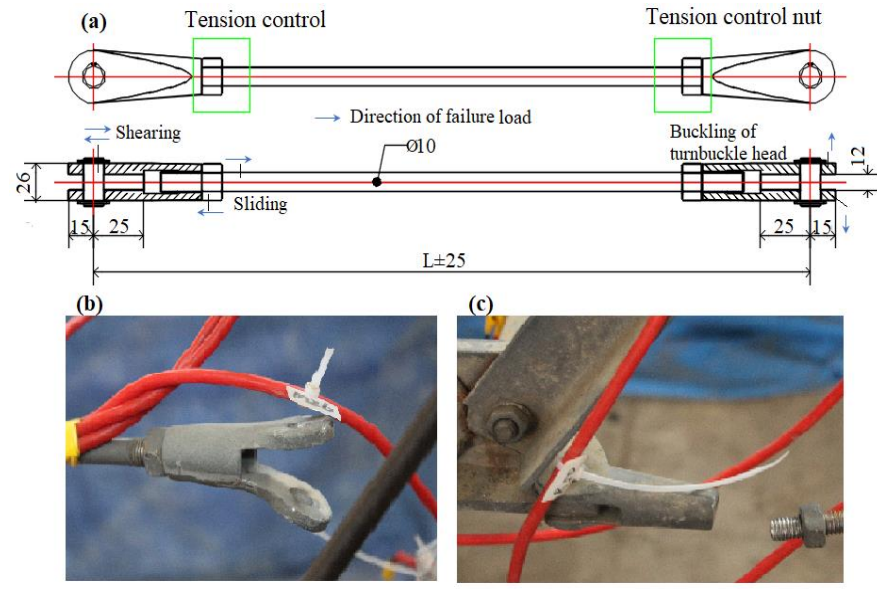

Fig. 7 Failure mode of the cable member: (a) Diagram of the cable member with direction of failure load, (b) Shear and buckling failure of turnbuckle joint and (c) slide failure of turnbuckle joint

\subsection{Validation of Model}

The result of the experiment provided the measured axial forces for a series of failed structural members. The shear and sliding resistance of the joint was designed as $75 \%$ of the cable ultimate load, representing the failure load of the cable member during the experiment. From both experimental and analytical models, the values of axial forces and displacement for the broken cable members were obtained. The values of axial forces in the mid-span cable members after redistribution and the joint failure modes taken from both the experimental and analytical are presented in Table 2. As shown in Table 2, the strain rate of 0.03 to 0.05 was applied as the break limit of the cable members according to the measured value from the experiment. The variation of the force between the experimental and analytical against the limit axial load $\left(S_{\text {lim }}\right)$ can be observed in Fig. 8 . While the maximum displacement for the corresponding broken cable members are presented in Table 3. In this study, the values obtained from both experimental and numerical models are in close agreement with the recorded ratios of experimental/analytical ranging from 0.81 to 0.95 as shown in Tables 2 and 3 .

As earlier noted, the transient response time of the experimental models is in the limit of $1 \mathrm{~s}$. According to the analytical results, the peak displacement for each models was reached within $0.1 \mathrm{~s}$ as shown in Fig. 9. For example, the peak displacement of model $\mathrm{ST} 1.8 \mathrm{~m} / 1$ was at a time duration of $0.06 \mathrm{~s}$. It was observed that the sudden break of the cable member and the resulting redistribution of force brought about local deformation behavior at the region of failure which reflects buckling characteristic of the CSPS structure. It is generally observed that the maximum displacement is recorded at the mid-span of the structure. The transient force redistribution and displacement response for the sudden break of various members are subsequently presented.

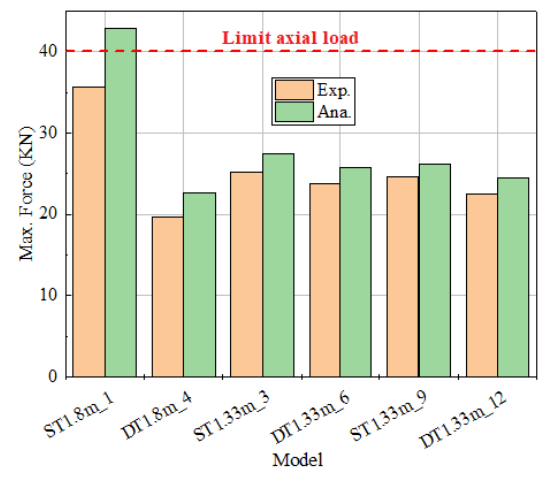

Fig. 8 Variation of axial force of mid-span cable after redistribution of force experimental and analytical models

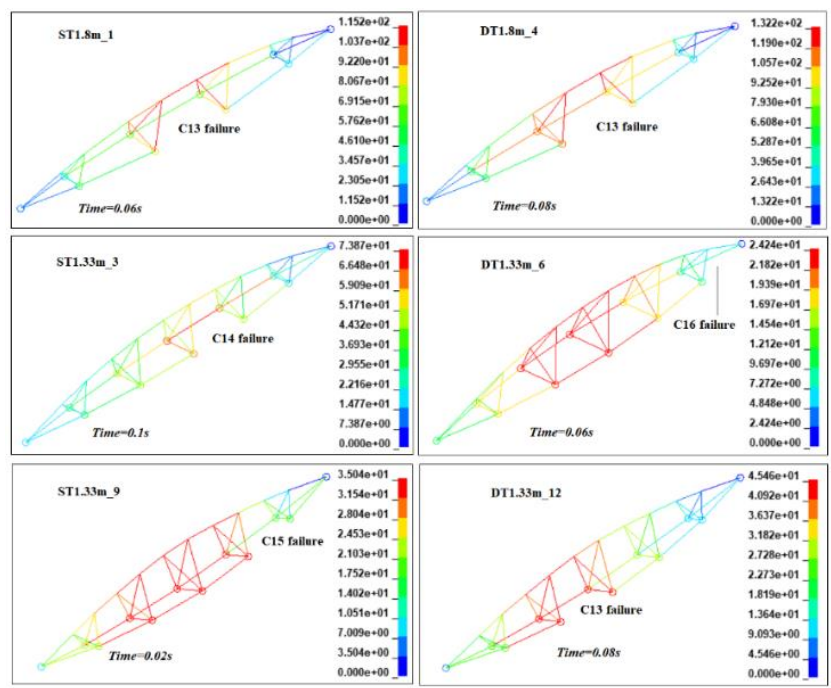

Fig. 9 Peak displacement (mm) of the analytical models 


\subsection{Effect of sudden failure of various cable members}

The break cases of the various cable members along the span are evaluated. Referring to Fig. 4, models ST1.8m/1 and DT1.8m/4 have 5 cases represented by the cable members $\mathrm{C} 11$ to $\mathrm{C} 15$ while models $\mathrm{ST} 1.33 \mathrm{~m} / 3$, DT $1.33 \mathrm{~m} / 6, \mathrm{ST} 1.33 \mathrm{~m} / 9$ and DT $1.33 \mathrm{~m} / 12$ have 6 cases represented by the cable member $\mathrm{C} 11$ to $\mathrm{C} 16$ along the spans. Following the numerical simulations, Fig. 10 presents the displacement responses of the CSPS models

ST1.8m_1

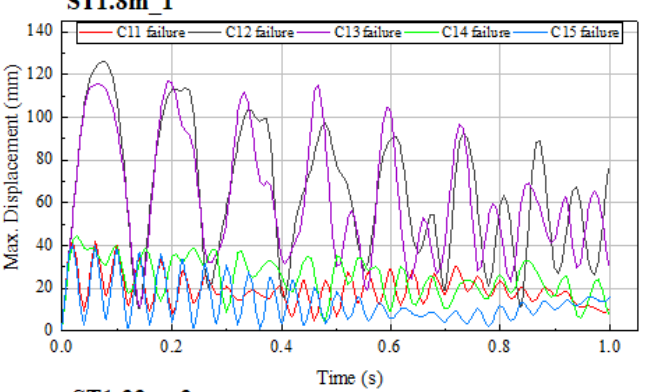

ST1.33m 3
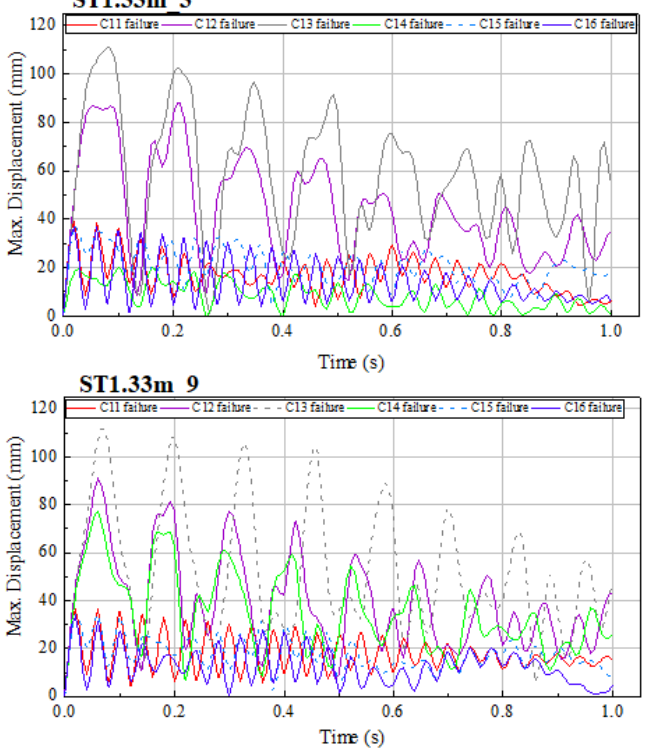

after different cable member removal. According to the results, the sudden break of cable member near the supports (cable members $\mathrm{C} 11$ and $\mathrm{C} 15$ for models ST1.8m/1 and DT1.8m/4, and C11 and C16 for the other models) induced a dissipated response, while the effect was more pronounced on the sudden break of the cable member near the roller support (i.e. cable member C11). Similar trend was recorded on all the models in which the sudden break of the mid-span cable yielded the highest vibratory responses.
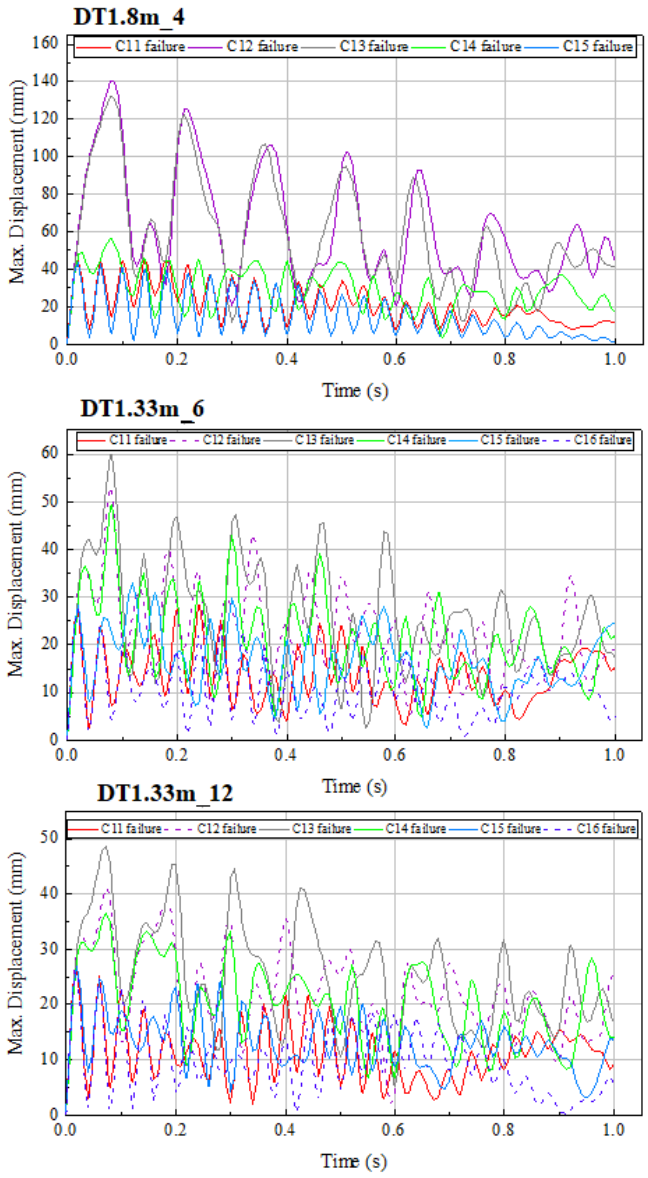

Fig. 10 Transient Displacement response considering the sudden break of various cable members

Table 2

Midspan cable force $(\mathrm{KN})$ of the CSPS Model after failure

\begin{tabular}{|c|c|c|c|c|c|c|}
\hline Model & Exp. & Ana. & Exp./Ana. & Strain rate of cable & Failed member & Mode of failure \\
\hline $\mathrm{ST} 1.8 \mathrm{~m} / 1$ & 35.7 & 42.9 & 0.84 & 0.05 & $\mathrm{C} 13$ & Sliding \\
\hline DT $1.8 \mathrm{~m} / 4$ & 19.6 & 22.6 & 0.86 & 0.05 & $\mathrm{C} 13$ & Shear \\
\hline $\mathrm{ST} 1.33 \mathrm{~m} / 3$ & 25.2 & 27.5 & 0.92 & 0.05 & $\mathrm{C} 14$ & Sliding \\
\hline DT $1.3 \mathrm{~m} / 6$ & 23.7 & 25.8 & 0.92 & 0.03 & $\mathrm{C} 16$ & Shear \\
\hline $\mathrm{ST} 1.33 \mathrm{~m} / 9$ & 24.6 & 26.1 & 0.94 & 0.05 & $\mathrm{C} 15$ & Sliding \\
\hline DT $1.33 \mathrm{~m} / 12$ & 22.5 & 24.4 & 0.93 & 0.05 & $\mathrm{C} 13$ & Sliding \\
\hline
\end{tabular}

Table 3

Displacement at midspan of the CSPS Model

\begin{tabular}{|c|c|c|c|c|c|c|c|c|c|}
\hline \multirow[t]{2}{*}{ Model } & \multicolumn{3}{|c|}{ Longitudinal (mm) } & \multicolumn{3}{|c|}{ Lateral (mm) } & \multicolumn{3}{|c|}{ Vertical (mm) } \\
\hline & Exp. & Ana. & Exp./Ana. & Exp. & Ana. & Exp./Ana. & Exp. & Ana. & Exp./Ana. \\
\hline $\mathrm{ST} 1.8 \mathrm{~m} / 1$ & 17.7 & 19.8 & 0.89 & 118.5 & 141.4 & 0.84 & 93.7 & 107.3 & 0.87 \\
\hline DT $1.8 \mathrm{~m} / 4$ & 16.1 & 17.7 & 0.91 & 99.8 & 123.0 & 0.81 & 75.9 & 91.7 & 0.83 \\
\hline $\mathrm{ST} 1.33 \mathrm{~m} / 3$ & 25.6 & 26.4 & 0.97 & 4.5 & 5.2 & 0.87 & 7.3 & 8.7 & 0.84 \\
\hline $\mathrm{DT} 1.3 \mathrm{~m} / 6$ & 18.1 & 20.35 & 0.89 & 3.5 & 4.1 & 0.85 & 6.1 & 7.5 & 0.81 \\
\hline $\mathrm{ST} 1.33 \mathrm{~m} / 9$ & 11.4 & 13 & 0.88 & 18.9 & 20 & 0.95 & 38.6 & 40.2 & 0.96 \\
\hline $\mathrm{DT} 1.33 \mathrm{~m} / 12$ & 9.5 & 10.5 & 0.91 & 16.8 & 18.2 & 0.92 & 31.4 & 34.7 & 0.91 \\
\hline
\end{tabular}


The evaluated results of the axial force redistribution is presented in Fig. 11. The results showed that all the cable members experienced vibratory response on the sudden break of a memeber, and the peak force during redistribution occurred when the mid-span cable is broken. Due to stiffening effects on models with double tube pipe and additional truss module, the force amplitude aggravated by the sudden break of cable member was attenuated. Similarly, in models with adjustment of the depth to span parameter
(ST1.33m/9 and DT1.33m/12) the force amplitude aggravated by the sudden break of cable member was attenuated. Generally, larger force amplitudes are recorded on cable members at the mid-span as compared to the members nearer to the supports. It is thus noted that the force variations of the members were associated to their positions as shown in the recorded results. It can be stated that the sudden break of mid-span cable member results in the increase of tensile load beyond the limit load.
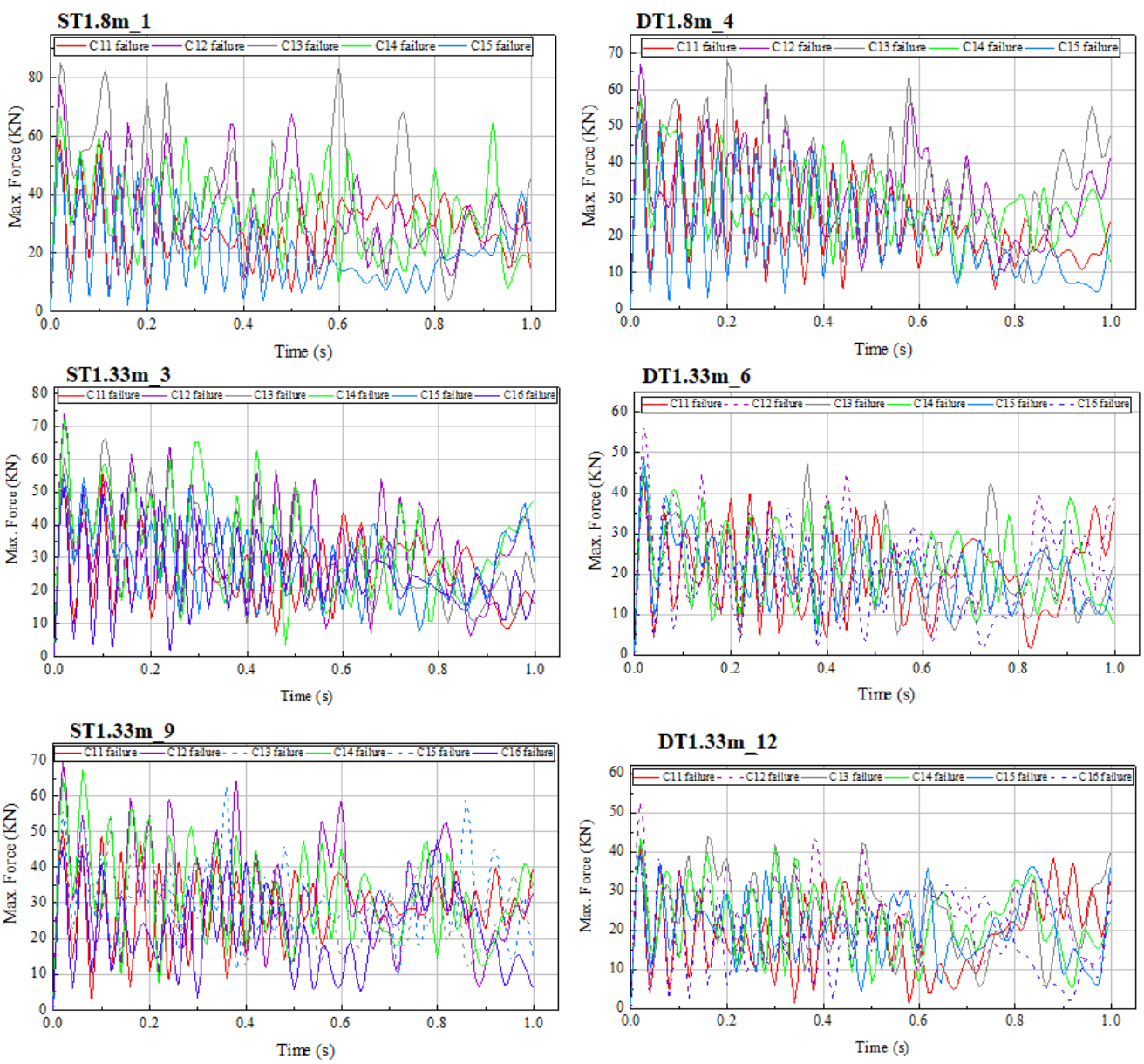

Fig. 11 Transient force response considering the sudden break of various cable members

The comparison of cable forces of implicit static loading before sudden break and corresponding explicit dynamic response after sudden break of the cable members are both presented in Fig. 12. As observed in Fig. 12(a), the internal forces in the cable members were kept within the limit of the axial force $\left(S_{\text {lim }}\right)$. However, in Fig 12(b) there was a rise in the internal forces in the cable members above $S_{\text {lim }}$ after the sudden break. Before the sudden break of a member, energy is conserved in the system and was released after the sudden break, thereby propagating transient vibratory response to achieve the redistribution of internal forces in the structure as shown in Fig. 11. Evaluation of the result of the variation of vibratory response of the explicit models and the sudden increase beyond implicit static $S_{\text {lim }}$ is attributed to the process of redistribution after the sudden break of a cable member. This phenomenon is only verifiable with the aid of explicit solver which can offer effective and precise solution to sudden break. It can be seen that the sudden break of any cable member for each CSPS model resulted in transient vibratory response with varying peaks. As earlier presented in Fig. 8, the internal force dissipated immediately the redistribution was achieved. For the cable members situated at the supports, there was internal forces increase with attendant vibratory response but with moderate effects compared to cable members away from the supports. The result also showed that the attenuation of the cable internal force increases from CSPS model ST1.8m/1 to DT $1.33 \mathrm{~m} / 12$ during break of any cable member. It is thus seen that the model DT $1.33 \mathrm{~m} / 12$ offered more resistance to the vibratory effect of sudden break of the CSPS members. The reduction in the peak displacement and dissipation of internal forces from ST1.8m/1 to DT $1.33 \mathrm{~m} / 12$ suggested that the modified structure offered more support against collapse. However, the results further suggested that the critical cable members are situated around the mid-span.
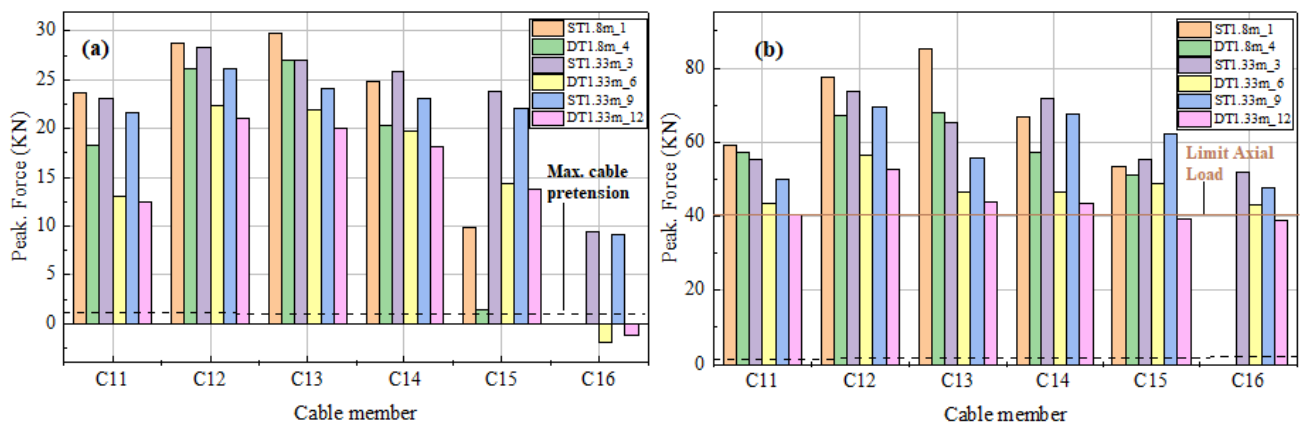

Fig. 12 Variation of peak cable force along the length of the structure: (a) during static loading and (b) considering sudden break of a member 


\section{Result of parametric evaluation}

The preceding discussion focused on the evaluation of dynamic effects of the modified CSPS model based on the various parameters. The results showed that the depth modification design parameters provide improved collapse resistance against sudden break of a cable member of the CSPS structure. Consequently, a comparison of the collapse resistance offered by member section parameter including the cable $(r-x c)$, truss $(t-x t)$ and pipe $(t-x p)$ of the models are evaluated considering the sudden break of critical cable member of model ST $1.8 \mathrm{~m} / 1$.

Comparison of the effect of the varying parameters of CSPS model under the sudden break of a critical member offered unique responses. Fig. 13 shows transient vibratory curves of the cable with depth modification parameters. The results suggest that the collapse resistance capacity is improved with modification of the pipe, the number of truss modules and the curvature of supporting cables. This is seen in the displacement response in Fig. 13(a) which suggests model DT1.33m/12 with combined modifications of the pipe, truss and curvature offers improved vibratory response. Similarly, the axial force redistribution as shown in Fig. 13(b) indicates dissipation of internal forces from the highest in model ST1.8m to lowest level in model DT $1.33 \mathrm{~m} / 12$. When double tube is applied in lieu of a single tube pipe member, the collapse resistance of the structure improves. However, it is yet to be concluded that the use of double tube is an improvement strategy as against the use of pipe thickness parameter. Thus, it is imperative to further evaluate the influence of various pipe thickness on the structure. Subsequent paragraphs deal with the parametric influence of the thickness of the members.
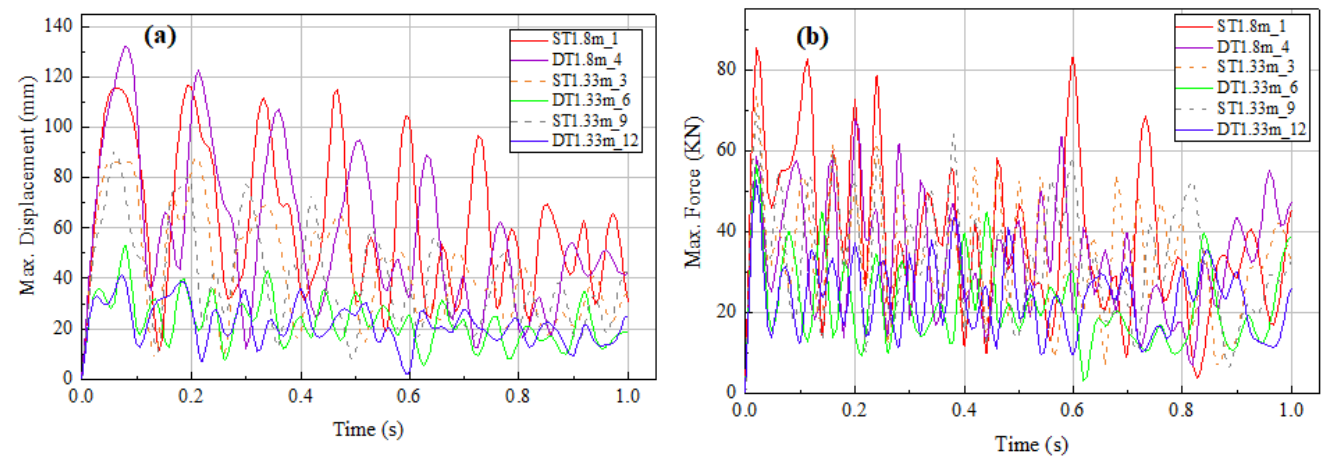

Fig. 13 Transient vibratory response of the CSPS models: (a) Displacement and (b) Axial force
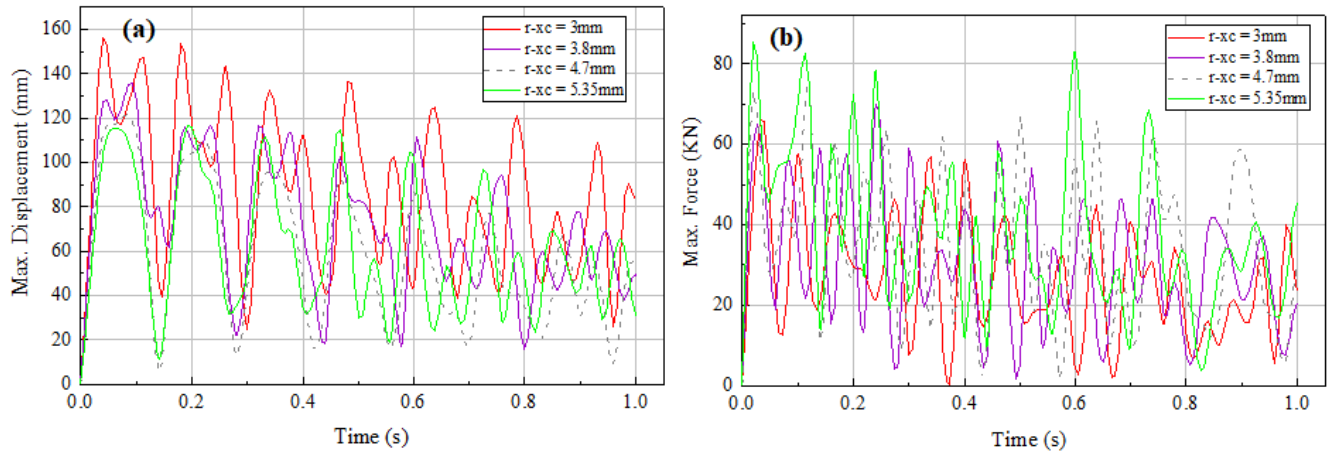

Fig. 14 Transient vibratory response of the cable parameters: (a) Displacement and (b) Axial force

Fig. 15 depicts the influence of the pipe parameter, $t-x p=2,3$ and $3.3 \mathrm{~mm}$ as well as DT $t-x p=3$ in improving the collapse resistance of the structure. The results also suggest that with higher values of $t-x p$ the structure offers more collapse resistance. As shown in Fig. 15(a), the largest displacement was recorded when the value of $t-x p$ is 2 and got dissipated when the value increased to 3.5. Similarly, the axial force increases, offering higher collapse resistance with higher values of $t-x p$ parameters as shown in Fig. 15(b). If a close look is taken at the values of $D T t-x p=3$ in both the displacement and axial force responses, it is once again verified that the application of a double tube pipe member, although increased the bearing capacity of the structure, offers improved collapse resistance by combined action with other measures. This was explained by the responses presented by model DT1.33m/12 in Fig. 13 .

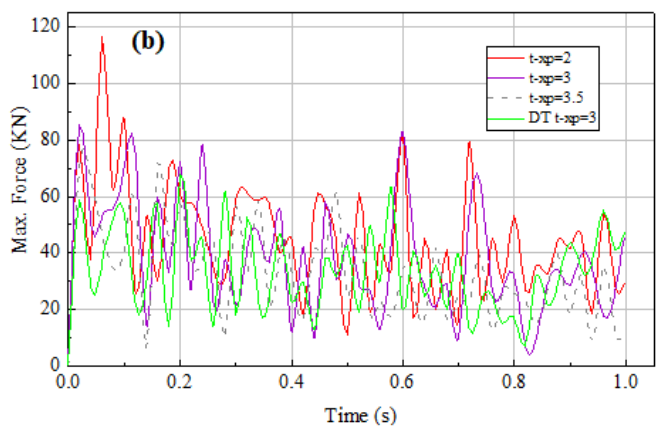

Fig. 15 Transient vibratory response of the pipe parameters: (a) Displacement and (b) Axial force 
Fig. 16 shows the displacement and axial force responses of the structure on the truss parameter $t-x t=2.4,3.0$ and $3.6 \mathrm{~mm}$. While adequate truss section is necessary for the overall performance of the structure, the results depict slight variation of both displacement and axial force response when a critical cable member is broken.
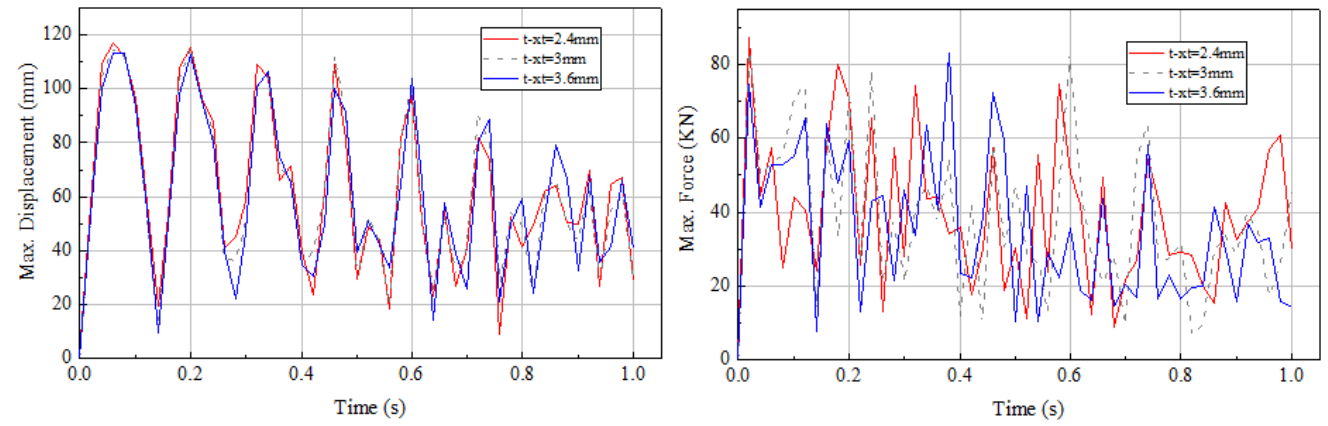

Fig. 16 Transient vibratory response of the truss parameters: (a) Displacement and (b) Axial force

The variations of peak axial force for various parameters after the sudden break of a critical member are presented in Fig. 17. Comparative assessment of the results of models revealed that the model with a double tube absorb the cable internal force more than the single tube models. It can be seen again that absorption increases from CSPS model ST1.8m/1 to DT1.33m/12 in the event of a sudden break of a critical cable member. Thus, the model DT1.33m/12 offered more resistance to the sudden break effect of the CSPS structure. The reduction in the peak axial forces from ST1.8m/1 to DT1.33m/12 in Fig. 17(a) further suggested that the modified structure offered more resistance to progressive collapse. However, the increase of the parameter $r$ - $x c$ depicted the ability to carry more axial force as shown in Fig. 17(b). Further evaluation of the parameter $t-x p$ revealed that the variation of axial force for the pipe thickness in the event of a sudden break of key cable member. Fig. 17(c) depicts a trend of absorption of internal forces with higher thickness of pipe and the use of double tube in lieu of single tube pipe members. Further evaluation with parameter $t-x t$ reals the variation of axial force as depicted in Fig. 17(d). It is obvious from the result that the section of truss member can be useful in improving the performance of the structure in the event of a sudden break. Overall, the parametric results placed emphasis on adequate member section when designing against the collapse of the CSPS structure.
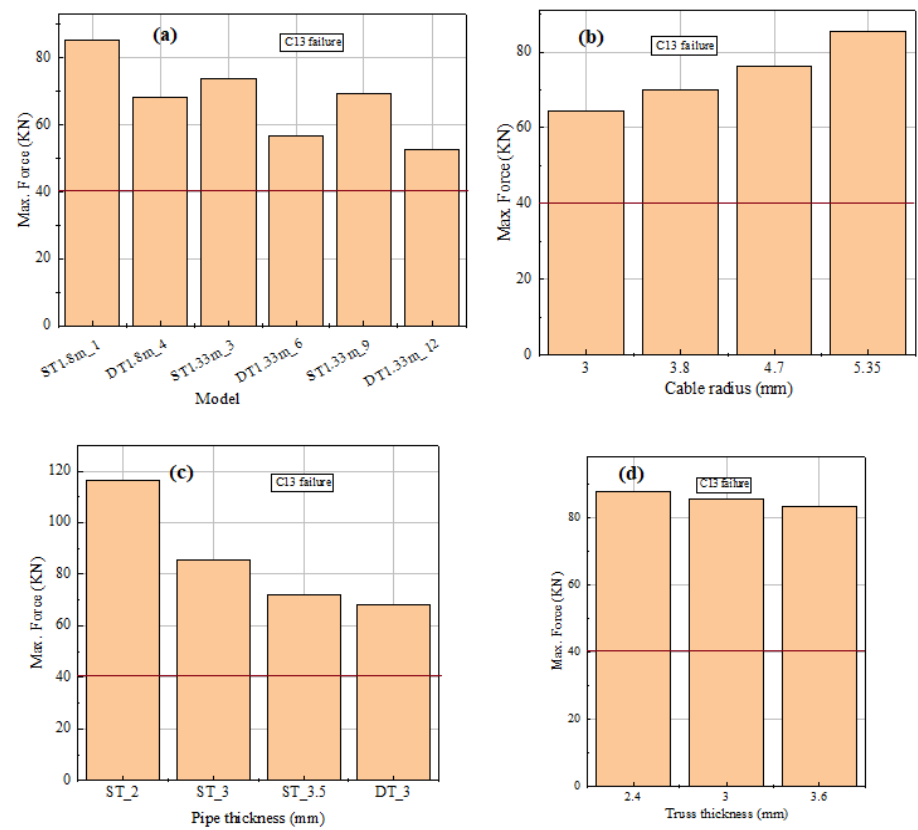

Fig. 17 Variation of peak axial force after the loss of a critical cable member: (a) CSPS models, (b) Cable parameters, (c) Pipe parameters and (d) Truss parameters

\section{Dynamic effect of sudden cable break}

\subsection{Dynamic increasing factor}

The method provided by most codes, standards and research work for the analysis and design against progressive collapse of most structures is to employ the dynamic increasing factor (DIF), commonly referred to as a dynamic amplifying factor, as a multiplier of the static. Other methods have been studied, including the method for evaluating the progressive collapse resistance of long-span single-layer spatial grid structures [29]. However, DIF reduces the rigorous process offered by the evaluation of dynamic event of the structure during the sudden break of a member. The dynamic increasing factor in every structural member is calculated separately considering all state variables $S$ [11]. Considering the sudden break of each of the cable members and the transient vibratory responses, the values of $S_{d y n}$ are determined as the peak dynamic response. From the static results obtained from implicit analysis, $S_{\text {stat }}$ is as the peak static response while the responses in the initial state $S_{0}$ are determined and subtracted from both $S_{d y n}$ and $S_{\text {stat }}$. Thus, DIF is obtained by comparing the dynamic and static responses expressed in equation (1) as follows:

$D I F=\frac{S_{d y n}-S_{0}}{S_{s t a t}-S_{0}}$

Given consideration to the specified design processes, the $D I F$ of 2.0 is provided to augment dynamic effect from the results of static analysis. However, the guide would not be suitable for inelastic structures. Thus, there is the requirement for further investigation due to nonlinear effect and flexibility induced by cable and truss members. Fig. 18 presents the calculated $D I F$ from the numerical results for the sudden break of various cable members of the CSPS models. Results revealed that the DIF data of the various members varied from the specified value of 2.0. The DIF results also revealed a dramatic effect of dynamic axial forces at the fixed supports with records of higher magnitudes above 2.0. This is attributed to the relatively smaller static forces arising from the application of double tube pipe members.

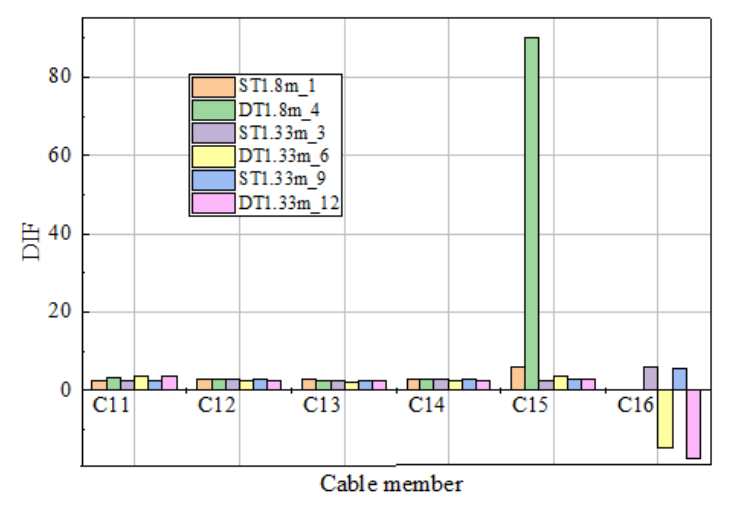

Fig. 18 Dynamic increasing factor $(D I F)$ after the loss of various cable member

In Fig. 19, the DIFs arising from a sudden break of a critical member for the parametric analysis of the models are presented. Fig. 19(a) shows the variation of $D I F$ for the various models. The results further verify the influence of the modification of the CSPS model. As reorded, all the modified models relatively reduce the value of the DIF. It is observed that with an increase in the absorption of axial force from CSPS model ST1.8m/1 to DT $1.33 \mathrm{~m} / 12$ in the event of a sudden break of a critical cable member, there is 
a corresponding decrease in the value of DIF. The trend slightly differs on model DT $1.33 \mathrm{~m} / 12$ which was presumed to have offered more resistance to the sudden break effect of the CSPS structure. However, in Fig. 19(b) with the larger value of parameter $r-x c$, the result depicts a rise in the DIF attributed to the increasing capacity to carry more axial force. Based on the evaluations above, it is obvious that the cable parameter exhibits internal force absorption due to the release of the most portion of its stored energy. With smaller value of $r-x c$, a relatively large tension, less vibratory response and low DIF values are recorded. The values of $D I F$ were also increased in the event of lower values of $S_{\text {stat }}$. Consequently, in the consideration of the sudden break of cable members of the CSPS structure, it is assumed that the DIF method offered more useful results for the vibratory responses of the pipe and truss member modifications.

The $D I F$ values of both the pipe and truss members parameters subject to sudden break of a critical member are also presented in Fig. 19. In Fig. 19(c), the decreasing values of $D I F$ arising from increase in the parameter $t-x p$ depicts an improvement in the collapse resistance. The modification with double tube in lieu of single tube pipe members is obviously useful in improving the collapse resistance in the event of hydraulic demand. In the context of $t$-xt, the variation of DIF depicted in Fig. 19(d) shows slight improvement in the collapse resistance due to the increase in section of truss member.

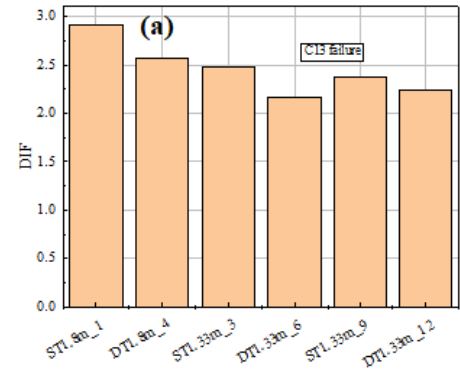

Model

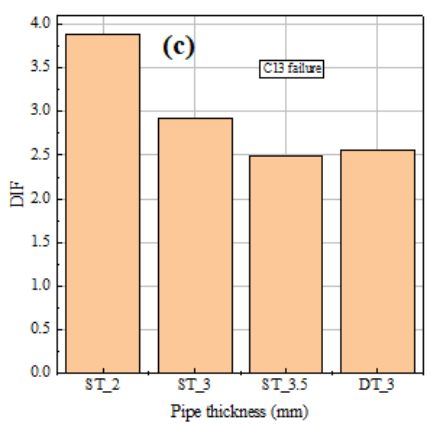

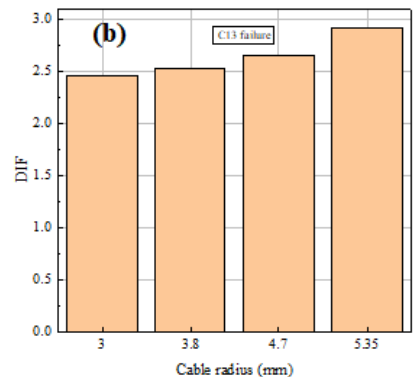

(d)

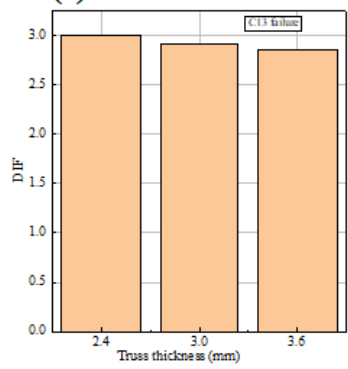

Fig. 19 Dynamic increasing factor $(D I F)$ after the loss of a critical cable member: (a) CSPS models, (b) Cable parameters, (c) Pipe parameters and (d) Truss parameters

\subsection{Revision of dynamic incearsing factor}

Since the application of DIF in the evaluation cable members is presumed to offer less suitable index for collapse resistance design of the CSPS structure, a revised index is hereby considered. Owing to the fact that the cable members are made of tension rods, the pretension varied from 0 to $0.9 \mathrm{KN}$ along the span amounting to less than $3 \%$ of the limiting axial load, thus, the application of $S_{0}$ in equation (1) was considered to be negligible. Thus, the DIF expression was modified and expressed as a dynamic factor $(D F)$ to account for the effect of the vibratory response and peak axial load during the sudden break of a cable member. $D F$ is expressed in equation (2) as follows:

$D F=\frac{S_{d y n}}{S_{\text {stat }}}$

Fig. 20 presents the values $D F s$ from the numerical results for the sudden break of various cable members of the CSPS models. The recorded $D F$ values of the various members are relatively closer to the specified $D I F$ value of 2.0. The results also showed a dramatic effect of $D F$ for the values at the fixed support arising from the modified effect of the double tube pipe members.

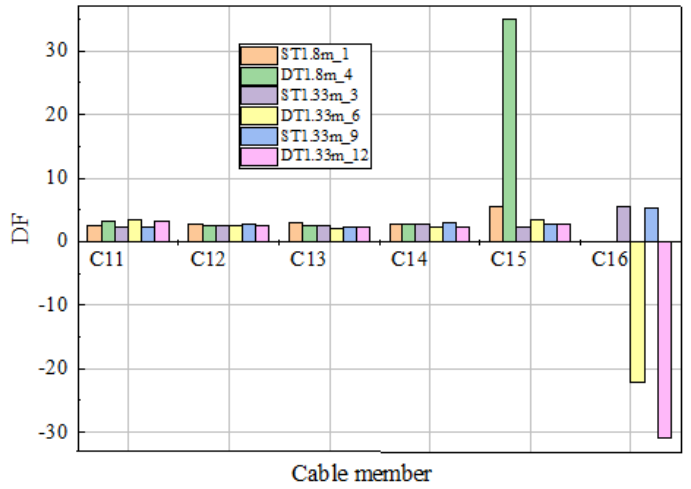

Fig. 20 Dynamic factor $(D F)$ after the loss of various cable member

Fig. 21 shows the DFs arising from a sudden break of a critical member for the parametric analysis of the models. In Fig. 21(a), the results show the effect of modified CSPS models on the values of $D F$. All the modified models relatively reduce the value of the $D F$. The $D F$ of other parameters also shows a similar trend with reduced index. In Fig. 21(b), larger value of parameter $r-x c$ results in a rise in the $D F$. Similarly, Fig. 21(c) shows the DF values of various $t-x p$ parameter, also depicting reduce index. Similar trends of reduced index is also seen in the results of $t$-xt parameters, as shown in Fig. 21(d).

It can be seen in Fig. 21(a) that all the DFs for the sudden break of any cable member along the span ranged from 2.4 to 5.5, except for the influence of double tube pipe members in which much greater values were recorded at the fixed supports. The fall in the DIF from St $1.8 \mathrm{~m} / 1$ to DT1.33m/12 once again suggested that the modified structure can offer improved resistance to progressive collapse. In comparison to $D I F$, it is deduced that the $D F$ offers a more realistic value for the evaluation of the vibratory response for the design of CSPS structure.

\subsection{Evaluation of incurred error}

It is noted that the $D I F$ and $D F$ defer from the specified value by most studies and codes in which the dynamic analysis is replaced by the results of simplified static analysis factored by a $D I F=2.0$. Considering the variation from the values, it is necessary to evaluate the error incurred by application of the simplified approach. Mozos utilized the empirical method of PTI in the parametric study on the dynamic response of cable-stayed bridges subjected to the sudden failure of a stay considering the bending moment of deck. The results which were evaluated as a function of the bending due to dynamic loads were obtained and finally expressed as a percentage of the envelope of the ultimate limit states for permanent and transitory loads [5]. In this study, the error is evaluated in the same model, as qualitative error $\left(E_{q}\right)$ according to the equation (3), taken as $E_{\text {stat }}$ in which the variation between the peak vibratory response and the static result factored by a $D I F=2.0$ is expressed as a percentage of the limit load $S_{\text {lim }}$.
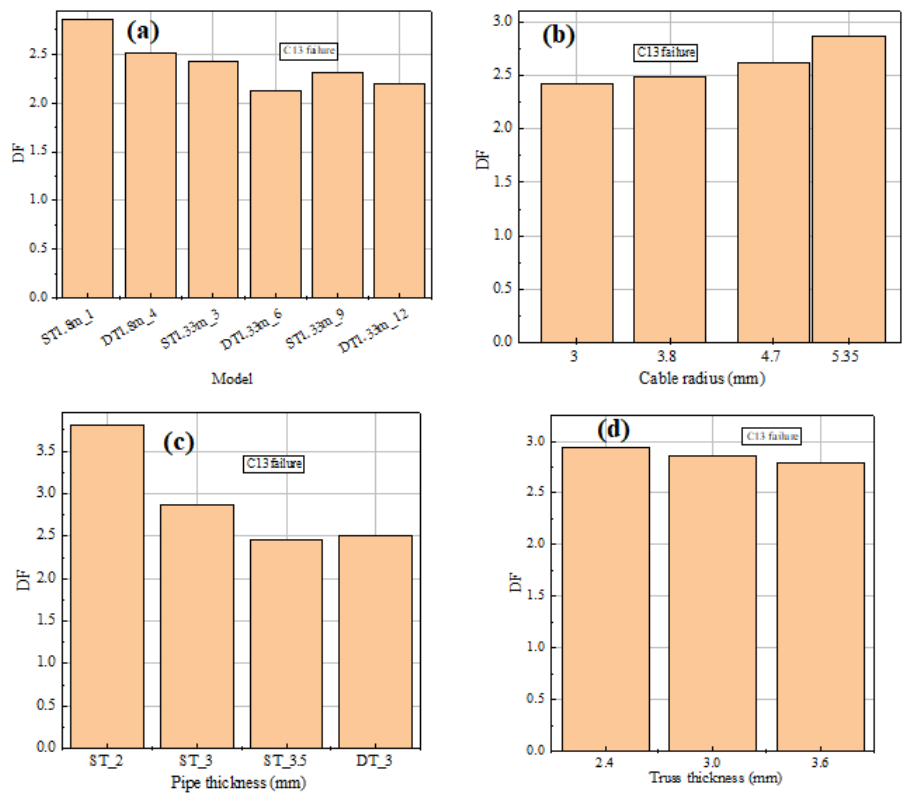

Fig. 21 Dynamic factor $(D F)$ after the loss of a critical cable member: (a) CSPS models, (b) Cable parameters, (c) Pipe parameters and (d) Truss parameters 
$E_{S t a t}=\frac{S_{d y n}-S_{D I F=2}}{S_{\lim }} \times 100$

However, the expression of $E_{\text {stat }}$ was used to evaluate the results of the implicit solver. The evaluation using $E_{\text {stat }}$ is considered unsuitable for the present study, thus, the revision of error model is evaluated. To account for the dynamic effect using the explicit solver, the revised error is expressed as $E_{d y n}$ in which static result factored by a $D I F=2.0$ is expressed as a percentage of the peak vibratory response $S_{d y n}$ as shown in equation (4).

$E_{d y n}=\frac{S_{d y n}-S_{D I F=2}}{S_{d y n}} \times 100$

The comparative evaluation of the results of both $E_{\text {stat }}$ and $E_{d y n}$ revealed higher error margin from the $E_{\text {stat }}$. Fig. 22 depicts the case of a sudden break of cable members along the span of the models. As shown is Fig. 22(a), the $E_{\text {stat }}$ increases at the fixed support with values of $84.4 \%, 120.8 \%, 82.3 \%$, $117.7 \%, 73.5 \%$ and $103.6 \%$ of the respective models. Comparative evaluation of $E_{d y n}$ in Fig. 22(b) reveals drop in the values to $63.2 \%, 94.3 \%, 63.7 \%$,
$109.0 \%, 61.8 \%$ and $106.5 \%$ respectively. The higher values recorded at the fixed support cables C5 and C6 can be attributed to the limited vibratory response.

Fig. 23 shows the results of the parametric study of a sudden break of a critical member. Fig. 23(a) presents the error variations corresponding to the effect of the modified models. Except for the modified models, the error dropped from $64.2 \%$ to $30.1 \%$. The results also showed marginal drop at models DT1.33m/12 and DT1.33m/6. Thus, the model ST1.8m/1 will have a larger error compared to the others. Fig. 23(b) shows that by increasing the parameter $r$ - $x c$ of $\mathrm{ST} 1.8 \mathrm{~m} / 1$, the error increases in a nonlinear pattern. Conversely, increasing the parameter $t$ - $x p$ cause decrease in the error as shown in Fig. 23(c). Furthermore, increasing the parameter $t$ - $x t$ causes decrease in the error in a linear pattern as shown in Fig. 23(d).

Based on all the evaluated $D F$ values in 5.1 and 5.2 and the corresponding errors when compared to the static amplified by a factor of 2 , the dependable value of the $D I F$ is in the range of 2.4 to 3.8 except for the extreme values recorded by the models with DT pipe members. These values can be increased by considering a factor of safety during the design process of the CSPS structure. In practice, DIF of 1.2 is recommended, however, this study recorded increased values in the range of 2.88 to 4.56 .
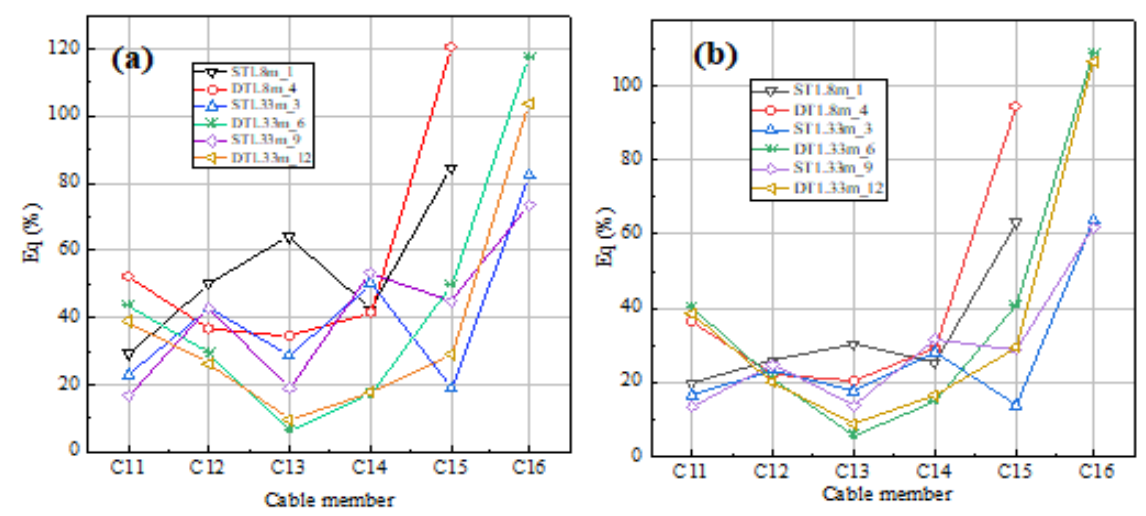

Fig. 22 Error incurred considering DIF=2 for static load as against the proposed Dynamic factor due to the sudden loss of

various cable member of the CSPS models: (a) $E_{s t a t}$ and (b) $E_{d y n}$
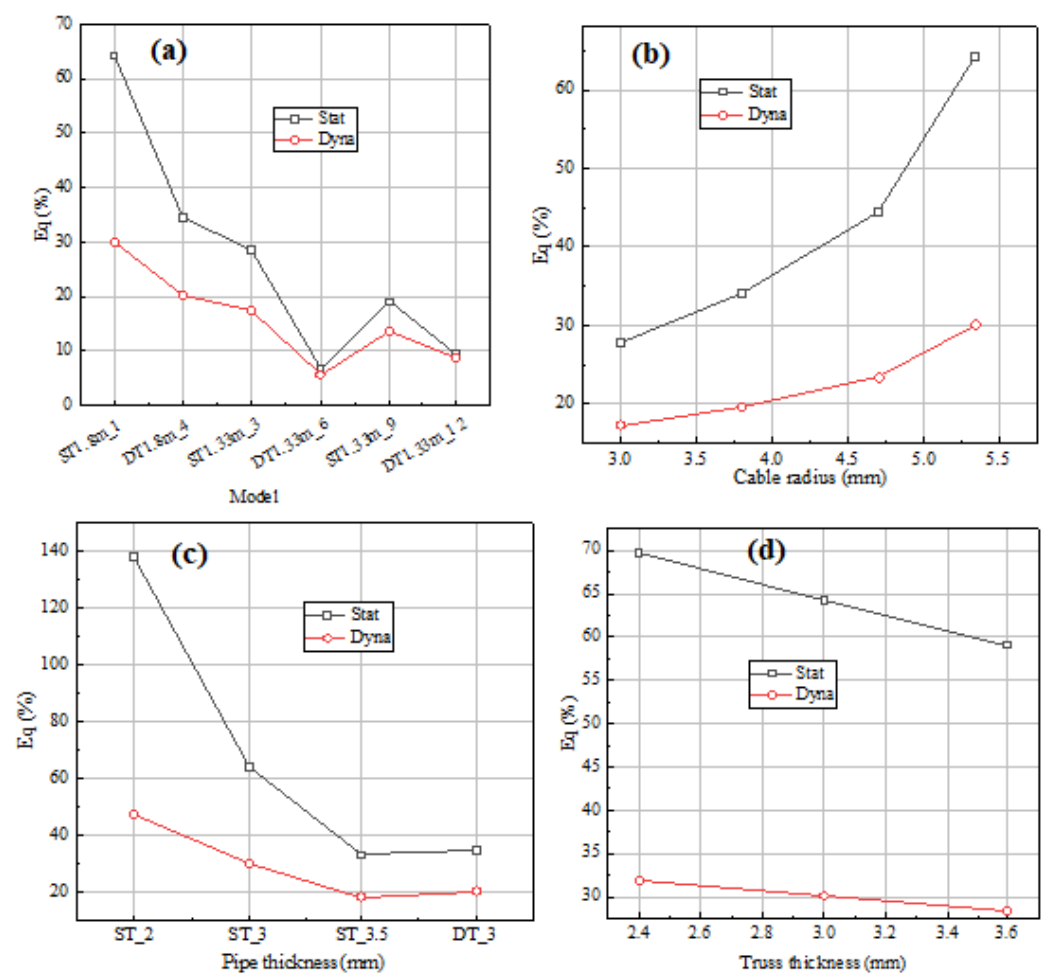

Fig. 23 Error incurred considering DIF=2 for static load as against the proposed Dynamic factor due to the sudden loss of a critical cable member: (a) CSPS models, 


\section{Conclusion}

This study presented a parametric study on the response of cable-supported pipe system (CSPS) subjected to the sudden break of cable members. The study covered the collapse resistance offered by the modification of the geometric features such as the number of truss modules, mid-span depth, single and double tube pipe members, as well as the cross-section of cable, pipe and truss members of the CSPS structure. The models were investigated using the limit axial load, $S_{\text {lim }}$ established from static loading experimental processes leading to the sudden break of the turnbuckle joints. The loading test was used to establish the break duration and vibratory response phenomenom. After verifying the explicit analysis with the loading test results, the models were subjected to further numerical study, and the results were further evaluated by comparison with the simplified static analysis in which the recommended $D I F$ value of 2.0 was factored to the static load. The objectives of the study are to establish suitable dynamic indices for cable members and qualitatively obtain the residual of value the CSPS structure after the redistributed axial force in the event of a sudden break of a cable member in the design process of the CSPS structure. The main conclusions of the study are:

1) Once sudden break of cable member occurs in a CSPS structure, vibratory response process and redistribution of internal forces are experienced. The results of the response process of the experiment suggested that the numerical procedure can be considered as an effective tool for the prediction of the sudden break, vibratory response and internal force redistribution of the CSPS structural members. In the response, the sudden break of a cable member caused large displacement which obviously reflected in the obtained results of both the analytical and experimental models. This displacement is more pronounced in the $\mathrm{ST} 1.8 \mathrm{~m} / 1$ and it is attenuated in the modified models.

2) The sudden break of cable member and the process of internal force redistribution apparently brought about a vibratory response on the structure. This transient vibratory response could be significant, and detrimental to the residual strength of the cable members. The condition may also influence the behaviour of the truss and pipe members. The dynamic effect must be considered in structural design.

3) In other to realistically portray the behaviour of the structure in the design or analysis of CSPS structures, the dynamic effect due to sudden break of a cable member is necessary. Two indices were evaluated to establish the collapse mitigation strategy, the dynamic increasing factor and a modified version, termed a dynamic factor. From the results of the analytical processes, while the $D I F$ evaluation method can be utilized for members other than the cables as recommended in other studies[11], the $D F$ is suggested in this study to offer a more realistic evaluation index for cable members. However, due to the different vibratory responses among members, both $D I F$ and $D F$ will be useful in the practical design of CSPS structure.

4) The study of the sudden break of cable-supported structures using static load is considered having a relatively localized dynamic effect which occurred around the region of the broken member and the region with higher internal force redistribution. On the contrary, applying dynamic load such as wind and seismic could produce a different result of the redistributed internal force in the structure. Thus, the influence of applied load and the resulting internal force redistribution in the event of a sudden break of a cable member should be verified before the application of $D I F$ and $D F$ for the design of the CSPS structure.

5) Considering that the method suggested by other studies and the PTI in the evaluation of qualitative error account more on the static effect, a modified expression was proposed to account for the dynamic effect. The proposed method utilized the difference between vibratory response and the static response factored with a DIF of 2.0 expressed as a percentage of vibratory response. The error margins of the results from the two methods suggested unrealistic results, thus portrayed limitation of the implicit solver for the evaluation of the sudden break of cable member of the CSPS structure. However, suggested method using DIF of 2.0 is considered unsuitable for cable members of the CSPS structure due to the recorded margin of error in both methods. This forms a basis for further research.

\section{Acknowledgements}

This study is supported by Hebei Province Full-time Top-level Talents Introduction Project (Grant No. 2020HBQZYC013), and National Key Research and Development Program of China (Grant No. 2018YFC0705500, 2018YFC0705504). Their financial support for this work is highly recognized.

\section{Reference}

[1] D. A. Christensen and R. D. McGee, "Irrigation drive unit," U.S. Patent No. 7,229,032. 12 Jun. 2007.

[2] N. J. Gimsing and C. T. Georgakis, "Cable supported bridges: Concept and design," John Wiley \& Sons, 2011

[3] M. P. Agwoko, Z. Chen, and H. Liu, "Experimental and Numerical Studies on Dynamic Characteristics of Long-Span Cable-Supported Pipe Systems," International Journal of Steel Structures, 2020/11/13 2020.

[4] Z. Chen, "Suspen-dome Structure," ed: Science Press, Beijing, China, 2010

[5] C. Mozos and A. Aparicio, "Parametric study on the dynamic response of cable stayed bridges to the sudden failure of a stay, Part I: Bending moment acting on the deck," Engineering Structures, vol. 32, no. 10, pp. 3288-3300, 2010.

[6] C. Mozos and A. Aparicio, "Parametric study on the dynamic response of cable stayed bridges to the sudden failure of a stay, Part II: Bending moment acting on the pylons and stress on the stays," Engineering Structures, vol. 32, no. 10, pp. 3301-3312, 2010.

[7] M. Wolff and U. Starossek, "Cable loss and progressive collapse in cable-stayed bridges," vol. 5, no. 1, pp. 17-28, 2009.

[8] X. Wang, Z. Chen, Y. Yu, and H. Liu, "Numerical and experimental study on loaded suspendome subjected to sudden cable failure," vol. 137, pp. 358-371, 2017.

[9] B. Asgarian, S. D. Eslamlou, A. E. Zaghi, and M. Mehr, "Progressive collapse analysis of power transmission towers," Journal of Constructional Steel Research, vol. 123, pp. 31-40, 2016.

[10] C. M. Mozos and A. C. Aparicio, "Numerical and experimental study on the interaction cable structure during the failure of a stay in a cable stayed bridge," Engineering Structures, vol. 33, no. 8, pp. 2330-2341, 2011/08/01/2011.

[11] P. T. I, Guide specification, "Recommendations for Stay Cable Design, Testing and Installation," Post-tensioning Institute Committee on Cable stayed bridge, Fifth Edition, DC45.1-12, 2007

[12] Y. Zhou and S. Chen, "Time-progressive dynamic assessment of abrupt cable-breakage events on cable-stayed bridges," vol. 19, no. 2, pp. 159-171, 2014

[13] Y. Zhou and S. Chen, "Numerical investigation of cable breakage events on long-span cable-stayed bridges under stochastic traffic and wind," Engineering Structures, vol. 105, pp. 299-315, 2015.

[14] Y. Zhou and S. Chen, "Framework of nonlinear dynamic simulation of long-span cable-stayed bridge and traffic system subjected to cable-loss incidents," Journal of Structural Engineering, vol. 142, no. 3, p. 04015160, 2016.

[15] Z. H. Chen, X. X. Wang, H. B. Liu, and Y. J. Yu, "Failure test of a suspendome due to cable rupture," vol. 15, no. 1, pp. 23-29, 2019

[16] B. S. 5950, "British Standards Institution: Structural use of steel work in buildings, Part1 Code of practice for design - Rolled and welded sections." London(UK); 2002.

[17] D. Stevens, B. Crowder, D. Sunshine, K. Marchand, R. Smilowitz, E. Williamson and M. J. Waggoner, "DoD research and criteria for the design of buildings to resist progressive collapse," vol. 137, no. 9, pp. 870-880, 2011.

[18] K. Marchand, A. McKay, and D. J. Stevens, "Development and application of linear and non-linear static approaches in UFC 4-023-03," in Structures Congress 2009: Don't Mes with Structural Engineers: Expanding Our Role, 2009, pp. 1-10.

[19] J. O. Malley, C. J. Carter, and C. M. Saunders, "Seismic design guidelines and Provision for steel-framed buildings: FEMA 267/267A and 1997 AISC Seismic Provisions," Earthquake spectra, vol. 16, no. 1, pp. 179-203, 2000.

[20] M. S. Zarghamee, S. Bolourchi, and D. W. Eggers, "NIST NCSTAR 1-6D: Federal Building and Fire Safety Investigation of the World Trade Center Disaster: Global Structural Analysis of the Response of the World Trade Center Towers to Impact Damage and Fire," in National Institute of Standards and Technology (US), 2005, no. NIST NCSTAR 1-6D: National Institute of Standards and Technology (US).

[21] W. G. Corley, "Lessons learned on improving resistance of buildings to terrorist attacks," vol. 18 , no. 2 , pp. $68-78,2004$

[22] H. S. Lew, R. Bukowski, N. J. Carino, and H. S. Lew, Design, construction, and maintenance of structural and life safety systems. US Department of Commerce, Technology Administration, National Institute of ..., 2005 .

[23] W. Xue and S. Liu, "Design optimization and experimental study on beam string structures," Journal of Constructional Steel Research, vol. 65, no. 1, pp. 70-80, 2009.

[24] GB, "Code for Design of Steel Structures" GB5017-2017; 2017.

[25] X. Wang, Z. Chen, Y. Yu, and H. Liu, "Numerical and experimental study on loaded suspendome subjected to sudden cable failure," Journal of Constructional Steel Research, vol. 137, pp. 358-371, 2017.

[26] S. Yan, X. Zhao, Y. Chen, Z. Xu, and Y. Lu, "A new type of truss joint for prevention of progressive collapse," vol. 167, pp. 203-213, 2018

[27] A.N.S.Y.S. Inc, "ANSYS LS-DYNA user's guide," ANSYS Inc, Pennsylvania, US, 2017.

[28] A. Documentation, "Release notes: Explicit dynamics analysis. Release 17.1," 2016.

[29] L. M. Tian, J. P. Wei, J. P. Hao, and X. Wang, "Method for evaluating the progressive collapse resistance of long-span single-layer spatial grid structures," Adv. Steel Constr, vol. 15 , no. 1 , pp. 109-115, 2019 